\title{
Broadly tunable multiwavelength fiber laser with Bismuth-oxide EDF using large effective area fiber.
}

\begin{abstract}
A multiwavelength laser comb using $2.49 \mathrm{~m}$ Bismuth-oxide erbium-doped fiber (Bi-EDF) with different lengths of large effective area fiber (LEAF) in a ring cavity configuration is realized. The Bi-EDF is used as the linear gain medium and LEAF is used as the non-linear gain medium for stimulated Brillouin scattering. Out of the four different lengths, the longest length of $25 \mathrm{~km}$ LEAF exhibits the widest tuning range of $44 \mathrm{~nm}(1576$ to $1620 \mathrm{~nm})$ in the Lband at $264 \mathrm{~mW}$ pump power and $5 \mathrm{~mW}$ Brillouin pump power. In addition, a total of 15 output channels are achieved with total average output power of $-8 \mathrm{dBm}$ from this laser structure. All Brillouin Stokes signals exhibit high peak power of above $-20 \mathrm{dBm}$ per signal and their optical signal-to-noise ratio of greater than $15 \mathrm{~dB}$.
\end{abstract}

Keyword: Brillouin pump; Brillouin Stokes; Erbium doped fibers. 